\title{
Measuring the absolute height and profile of the mesospheric sodium layer using a continuous wave laser
}

\author{
D. J. Butler ${ }^{1}$, R. I. Davies², R. M. Redfern ${ }^{3}$, N. Ageorges ${ }^{4}$, and H. Fews ${ }^{3, \star}$ \\ 1 Max-Planck-Institut für Astronomie, Königstuhl 17, 69117 Heidelberg, Germany \\ 2 Max-Planck-Institut für extraterrestrische Physik, Postfach 1312, 85741 Garching, Germany \\ 3 Experimental Physics Department, National University of Ireland, Galway, Ireland \\ ${ }^{4}$ European Southern Observatory, Alonso de Córdova 3107, Vitacura, Casilla 19001, Santiago 19, Chile
}

Received 29 November 2002 / Accepted 11 March 2003

\begin{abstract}
We have developed and tested a novel method, based on LIDAR, of measuring the height and profile of the mesospheric sodium layer using a continuous wave laser. It is more efficient than classical LIDAR as the laser is on for 50\% of the time, and so can in principle be used during laser guide star adaptive optics observations. It also has significant advantages over direct imaging techniques because it does not require a second telescope, is almost independent of the atmospheric conditions, and avoids triangulation problems in determining the height. In the long term, regular monitoring using this method would allow a valuable database of sodium layer profiles, heights, and return flux measurements to be built up which would enable observatory staff astronomers to schedule observations optimally. In this paper we describe the original experiment carried out using the ALFA laser guide star system at Calar Alto Observatory in Spain. We validate the method by comparing the LIDAR results with those obtained from simultaneous imaging from an auxiliary telescope. Models are presented of a similar system to be implemented in the Very Large Telescope Laser Guide Star Facility, which will enable the initial focus setting for the adaptive optics systems to be determined with an accuracy of less than $200 \mathrm{~m}$ on a timescale of $1 \mathrm{~min}$.
\end{abstract}

Key words. instrumentation: adaptive optics - instrumentation: miscellaneous - techniques: miscellaneous

\section{Introduction}

It is well known in the astronomical community that classical astronomical adaptive optics (AO) systems are only able to achieve their best performance over a few percent of the sky due to the scarcity of sufficiently bright reference (or guide) stars. Fortunately this severe limitation can be overcome by the use of a laser guide star (LGS), as suggested by Foy \& Labeyrie (1985). The principal advantage of a LGS is that it produces a bright reference source that can be pointed almost anywhere on the sky. Although a tip-tilt star is still needed, it can be further from the science target and much fainter, vastly increasing the sky accessible to adaptive optics systems. The main disadvantage of a LGS is that its proximity to the telescope means that it samples less of the atmospheric column seen by a natural star (the "cone effect", or "focal anisoplanaticism") resulting in poorer wavefront correction. For 8.0-m class telescopes which plan to attain the best correction possible with only a single LGS, the only reasonable choice is a sodium LGS generated by resonant excitation of sodium atoms in the Earth's atmosphere. In both the northern and southern hemispheres these

\footnotetext{
Send offprint requests to: D. J. Butler, e-mail: butler@mpia.de

* Current address: Marconi Communications, ONDATA GmbH, Germany.
}

are found only in the mesosphere at a height of $\sim 90 \mathrm{~km}$ (see She et al. 2000, and references therein). Atomic sodium abundance has been observed to vary seasonally by a factor of $3-4$, but is typically between about $2 \times 10^{13} \mathrm{~m}^{-2}$ around the solstices and $4-5 \times 10^{13} \mathrm{~m}^{-2}$ around the equinoxes at mid-latitudes (see Table 1).

Most of the sodium lasers which are planned for major astronomical observatories are designed to compensate for this so that they can be used at any time of year. For example, the Very Large Telescope (VLT) Laser Guide Star Facility (LGSF; Bonaccini et al. 2002) will provide a minimum of $10^{6}$ photons $\mathrm{m}^{-2} \mathrm{~s}^{-1}$ (equivalent to $\sim 1000$ photons per centroid measurement in a Shack-Hartmann wavefront sensor) even with a sodium column density of $2 \times 10^{13} \mathrm{~m}^{-2}$ during median atmospheric conditions. The launched laser power must then be at least $6 \mathrm{~W}$ (continuous wave, with four single frequency lines equally spaced within a $500 \mathrm{MHz}$ envelope). The laser used at the Keck Observatory is designed to meet similar requirements, and has a launch power of 15-20 W (pulsed, broadband spectrum; Pennington et al. 2002).

The occurrence of mesospheric atomic sodium is a natural phenomenon. Meteoric ablation in the upper atmosphere is thought to be the major contributor, with sudden short-lived increases of sodium density in localized regions of the layer 
known to occur (see Collins et al. 2002 and references therein for the various proposed explanations). These sporadic layers typically arise in thin layers about $1-3 \mathrm{~km}$ wide at altitudes between 90 and $105 \mathrm{~km}$. Since the increase in atomic density can be an order of magnitude more than the background mesospheric sodium density, a shift in the mean layer altitude could occur. Shifts as large as $400 \mathrm{~m}$ on timescales of 1-2 min have been observed by O'Sullivan et al. (2000). Such shifts would cause a LGS defocus (as well as, to a lesser extent, astigmatism and other higher order wavefront aberrations) to be sensed by a wavefront sensor. The rms wavefront error arising from uncorrected LGS defocus at a telescope with pupil size $D$ is given by $\sigma_{\lambda}=D^{2} \delta H / 16 \sqrt{3} H^{2}$ (Louarn et al. 2000) where $H$ is the height of the sodium layer $(\sim 90 \mathrm{~km})$. The corresponding Strehl loss is $S_{\text {loss }}=1-\exp \left(-\left(2 \pi \sigma_{\lambda} / \lambda\right)^{2}\right)$ where $\lambda$ is the wavelength at which improved angular resolution is required. For the 3.5-m at Calar Alto, where the measurements above were taken, a LGS defocus of $\delta H=400 \mathrm{~m}$ would introduce a wavefront error of $\sigma_{\lambda}=22 \mathrm{~nm}$ and a Strehl loss of $<0.5 \%$ at an observing wavelength of $2.2 \mu \mathrm{m}$. However, for an 8.0-m telescope we find $\sigma_{\lambda}=110 \mathrm{~nm}$ and the Strehl loss is $10 \%$, which cannot be ignored. Although focus changes resulting from tracking across the sky can in principle be calculated, those resulting from changes in the intrinsic height of the layer - which can exceed 1-2 km on longer timescales - cannot, and must instead be measured. At least three methods have been proposed to do this, namely direct imaging of the sodium plume; LIDAR (LIght Detection and Ranging); and averaging the LGS WFS focus error over 30-60 s.

The disadvantages of direct imaging of the plume are that an additional telescope is needed; height resolution depends on both seeing and distance from the main telescope; the pointing model is complex; and small errors can result in large errors in height determination (e.g., Michaille et al. 2000; and Sect. 3.4 of our paper). For example, if the monitoring telescope is $200 \mathrm{~m}$ from the main telescope, the LGS (at zenith) will appear as a plume $\sim 40^{\prime \prime}$ long. For typical seeing convolved with the intrinsic LGS size, the resolution of the profile will be limited to $\sim 300 \mathrm{~m}$. Additionally, an error in the pointing of only $1^{\prime \prime}$ results in a height error of $200 \mathrm{~m}$. The key advantage is that it can be performed independently of observations at the main telescope.

The principal advantage of LIDAR is the accurate height measurements, and their insensitivity to the ambient conditions. The disadvantage, however, is the extremely low duty cycle of the laser. Since the time between emitting a pulse and detecting the return flux is of order $1 \mathrm{~ms}$, the pulse rate of the laser must be slower than this, and in fact, typical rates are much less than $100 \mathrm{~Hz}$. This rules out the use of classical LIDAR during closed loop adaptive optics, unless a second laser is used.

A third option is to use the focus term from the WFS averaged over 30-60 s intervals. This should be zero, and any residual can be attributed to a change in height of the LGS. This would be an efficient on-line solution, but there could be problems with the initial focus setting of the WFS, particularly with curvature sensors. Additionally, the use of active optics during AO operation could induce further errors. Simulations for the case of the VLT (Bonnet 2001) suggest that the residual rms error should be only $35 \mathrm{~nm}$. However, this has been neither tested nor confirmed.

We have developed a method of modulating the beam of a continuous wave (cw) laser so that the duty cycle is $50 \%$ and only relatively small losses occur in transmission through the extra optics. The resulting average power remains high enough that the same laser can in principle be used simultaneously for both a LIDAR measurement and by an adaptive optics system. This is an important consideration since it is during observations with a LGS-AO system that these measurements are needed. Additionally, it is worth remarking that by making use of the small amount of light transmitted by AO system mirrors, which is usually unused, both LIDAR detection and the AO system can operate together without degrading AO performance; the design proposed for the VLT makes use of the $2 \%$ of light lost through one of the mirrors in the AO system. An experiment employing this technique was conducted with the ALFA (Adaptive optics with a Laser For Astronomy) AO-LGS system at the Calar Alto Observatory in Spain during 1999. The ALFA project was a joint development between the Max-Planck-Institutes for Astronomy (MPIA, in Heidelberg) and Extraterrestrial Physics (MPE, in Garching). It used a cw laser to generate a laser guide star, as this is very efficient in terms of return flux per Watt of launched laser power. A Shack-Hartmann WFS, which could be adjusted to the ambient seeing conditions by switching lenslet arrays, was able to measure the wavefront from either a natural or laser guide star.

We describe the principle of our sodium profiling method in Sect. 2, followed in Sect. 3 by a description of the observations, experimental set-up, and results, including a comparison of LIDAR results with direct imaging. Looking forward to the fast-approaching era of laser guide star operation at the VLT, we show in Sect. 4 the expected performance of the sodium layer profiler (Butler et al. 2002) to be installed at the Laser Guide Star Facility there. Finally, conclusions are drawn in Sect. 5.

\section{Principle of sodium profiling by LIDAR}

When using sodium fluorescence to measure the sodium density profile, it is important that we know the relation between the two quantities, which can depend on both the laser intensity and the sodium column density. If the laser intensity in the mesosphere is higher than $6 \mathrm{~W} \mathrm{~m}^{-2} \mathrm{MHz}^{-1}$ then saturation losses will reach $50 \%$. Since this is caused by the finite decay time of excited sodium atoms, it depends only on the laser intensity. For a $4 \mathrm{~W}$ laser like ALFA (cw, $10 \mathrm{MHz}$ bandwidth) modulated with $50 \%$ efficiency so that the maximum power launched during a LIDAR measurement is only $2 \mathrm{~W}$, a $1.5^{\prime \prime}$ spot would not saturate the sodium. The same is true for PARSEC (Rabien et al. 2002; Davies et al. 2002), the laser being built for the VLT. During a LIDAR measurement it will launch up to $8 \mathrm{~W} \mathrm{cw}$, with 4 single frequency lines within a $500 \mathrm{MHz}$ envelope. For such cases, we can be sure that the fluorescence and density profiles of the mesospheric sodium have a direct relation. Additionally, the sodium column density is low enough $\left(\sim 3 \times 10^{13} \mathrm{~m}^{-2}\right)$ that very little of the laser power is absorbed (only a few percent at zenith, and still less than $10 \%$ at a zenith 
Table 1. List (non-exhaustive) of sites where mesospheric sodium layer structure and/or abundance has been measured, and the type of laser used for the measurements.

\begin{tabular}{ccllll}
\hline \hline $\begin{array}{c}\text { Minimum } N(\mathrm{Na})^{a} \\
\left(10^{-13} \mathrm{~m}^{-2}\right)\end{array}$ & $\begin{array}{c}<N(\mathrm{Na})>^{a} \\
\left(10^{-13} \mathrm{~m}^{-2}\right)\end{array}$ & Latitude & Method & Laser & Reference \\
\hline$\sim 2$ & $\sim 3$ & $44^{\circ} \mathrm{N}^{1}$ & LIDAR & pulsed dye & Megie et al. (1977) \\
1.7 & $3.9^{b}$ & $41^{\circ} \mathrm{N}^{2}$ & LIDAR & pulsed dye & She et al. (2000) \\
2.3 & 4.3 & $40^{\circ} \mathrm{N}^{3}$ & LIDAR & pulsed dye & Papen \& Gardner (1996) \\
& & $37^{\circ} \mathrm{N}^{4}$ & LIDAR & cw dye & this paper \\
$\sim 2$ & 3.7 & $32^{\circ} \mathrm{N}^{5}$ & spectroscopy & cw dye & Ge et al. (1998) \\
2.6 & 3.6 & $29^{\circ} \mathrm{N}^{6}$ & imaging & cw dye & Michaille et al. (2001) \\
& & $20^{\circ} \mathrm{N}^{7}$ & LIDAR & pulsed dye & Gardner et al. (1991), Kwon et al. (1988) \\
& & $18^{\circ} \mathrm{N}^{8}$ & LIDAR & pulsed dye & Hecht et al. (1993), Collins et al. (2002) \\
2 & $\sim 4$ & $23^{\circ} \mathrm{S}^{9}$ & imaging & pulsed dye & Simonich et al. (1979), Clemesha et al. (1982) \\
\hline 2 & 3.6 & $30^{\circ} \mathrm{S}^{10}$ & imaging & cw dye & D'Orgeville et al. (2002) \\
\hline
\end{tabular}

\footnotetext{
${ }^{a}$ Minimum and mean sodium column densities, measured at zenith.

${ }^{b}$ Average of reported maximum and minimum $\left(6 \times 10^{-13} \mathrm{~m}^{-2}\right.$ and $1.7 \times 10^{-13} \mathrm{~m}^{-2}$ respectively).

${ }^{c}$ Spectroscopy of bright stars, to look at telluric sodium absorption, was used to determine Na abundance; this was compared to the laser guide star flux.

${ }^{1}$ Observatoire de Haute Provence; ${ }^{2}$ Fort Collins; ${ }^{3}$ Urbana; ${ }^{4}$ Calar Alto Observatory; ${ }^{5}$ Kitt Peak National Observatory; ${ }^{6}$ Observatorio de Roque de los Muchachos; ${ }^{7}$ Hawai ${ }^{i} ;{ }^{8}$ Arecibo Observatory; ${ }^{9}$ Sãn José dos Campos; ${ }^{10}$ Cerro Tololo Inter-American Observatory.
}

distance of $60^{\circ}$ ), so the laser intensity does not change much through the layer.

Classical LIDAR has been used to study the sodium layer for many years (see Table 1 and references in Clemesha 1995; Ageorges et al. 2000; Sica et al. 2002; and Collins et al. 2002). It consists of sending short laser pulses of the order of $0.1-1.0 \mu \mathrm{s}$ at a rate of a few $\mathrm{Hz}$ and collecting the returned photons in time bins of similar width to the outgoing laser pulses. By combining the returned photons from many laser "shots", the backscattering profile of the atmosphere is obtained; the signal-to-noise depends on the number of shots. Although the energy in each pulse is up to a few $\times 10 \mathrm{~mJ}$, saturation is avoided by illuminating a large area (20-200") of the sodium layer; such intrinsically pulsed sodium lasers are created by intra-cavity modulation (called Q-switching) - energy is stored up, and transmitted in a pulse. Additionally, pulse separations greater than $700 \mu$ s at zenith (and $1400 \mu$ s at a zenith distance of $60^{\circ}$ ) are required to avoid confusion over the location of the photon emission by overlapping of the return flux (both Rayleigh/Mie scattered from $\sim 15-25 \mathrm{~km}$ and fluorescent from $\sim 80-100 \mathrm{~km}$ ) from successive pulses. Such lasers cannot be used for simultaneous adaptive optics correction, which needs a compact laser spot on the sky and operates at frame rates up to $\sim 1 \mathrm{kHz}$.

The alternative would be intra- or extra-cavity modulation of an existing cw laser. However, for adaptive optics correction and sodium layer profiling in parallel, the low duty cycle of the classical LIDAR approach again provides too few return sodium layer photons, which would anyway be swamped by background noise from sky photons, dark noise, and stray photons. Importantly though, for the VLT sodium layer profiling system, the beam on the sky is focused into a $1.5^{\prime \prime}$ spot and observed through a small field aperture which cuts down the sky photon flux.

For laser modulation at high duty cycles, optical modulation is perhaps more convenient than the mechanical solution, a rotating chopper, as it allows a range of laser pulse widths and spacings. In acousto-optical modulation, for example, applying a radio-frequency signal to the modulator causes most of the laser light to be diffracted out of the straight-through beam into different directions. When the signal stops, the beam reverts to being straight-through. By using an aperture which allows only one of the beam directions to be transmitted (i.e. a laser beam is either all transmitted or all blocked), one can create digital On/Off pulses. Because a sequence of two or more high states is continually high, the pattern has a so-called "non-return to zero" format (see Fig. 1).

To maximize the sodium layer return flux, we have adopted this technique in order to launch a pseudo-random string of laser pulses with a "non-return to zero" format, providing a laser duty cycle of $50 \%$. The sequence can be repeated many times without pausing (as long as the time taken to complete one sequence is longer than the maximum round-trip time for a photon) and the return flux is recorded as a function of time in a series of consecutive measurement channels. Each time the sequence restarts, recording begins again in the first channel, adding to the counts already there.

If $S_{0}$ is the out-going laser pulse sequence then the returned stream of photons results from the convolution of $S_{0}$ with the sodium profile $N \otimes S_{0}$. The intrinsic sodium abundance profile, $N$, can be recovered from the data by cross-correlating it with the original pulse sequence if the auto-correlation of the sequence is very close to being a delta-function. Thus we find

$S_{0} \otimes\left(S_{0} \otimes N\right)=N$

For the ALFA experiment, we used a variation of this by oversampling, so although the pulses were $1 \mu$ s long we collected the returned photons in $0.25 \mu$ s time bins. Now to recover the sodium profile we consider the emitted laser pulse sequence as a sequence of impulses, $S_{1}$, four times longer in which each digit of $S_{0}$ is padded with zeros. If the profile of a pulse from the laser is denoted by $L$ then we can consider the emitted 


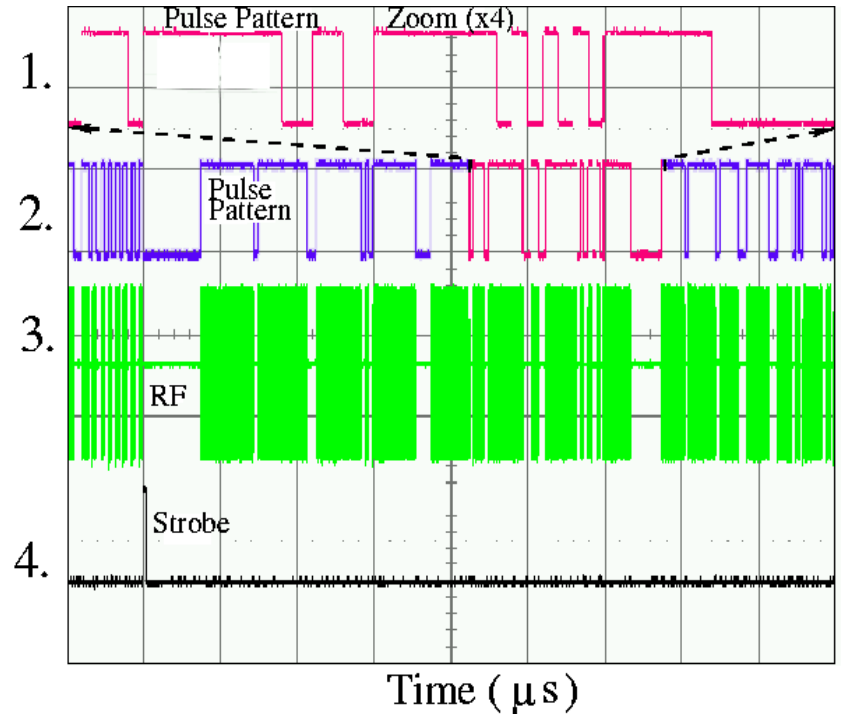

Fig. 1. Example section of a LIDAR electrical sequence generated by a pulse pattern generator and the associated radio frequency (RF) signal from the acousto-optical modulator driver. Channel 2: a $200 \mu \mathrm{s}$ snapshot of random $1 \mu \mathrm{s}$ TTL-pulses in "non-return to zero" format (at $2.0 \mathrm{~V}$ peak-to-valley). Channel 1: $4 \times$ zoom of channel 2 . Channel 3: $200 \mu$ s snapshot of the $40 \mathrm{MHz}$ RF signal. Channel 4: Strobe signal indicating the start of a sequence.

sequence as $S_{1} \otimes L$, and the returned flux is $S_{1} \otimes L \otimes N$. We can calculate the following cross-correlation

$S_{1} \otimes\left(S_{1} \otimes L \otimes N\right)=L \otimes N$

which gives the convolution of the sodium profile with the pulse profile. For the VLT LIDAR measurements, we will use Nyquist sampling and record the return flux in time bins equal to half of the pulse length; otherwise the method is similar.

Finally, a correction has to be made to this profile to compensate for the height at which each photon was scattered because the telescope mirror subtends a smaller solid angle for emission that originates higher in the atmosphere. This correction is applied by multiplying the profile data by the square of the height.

The shape of the pulse profile, and any height offset which might arise due to timing delays, can be found by carrying out the procedure with the telescope dome closed: this provides a single scattering layer at almost zero distance. As an example, the cross-correlated dome data from the ALFA LIDAR observations in Fig. 2 shows that there is a height offset of $270 \mathrm{~m}$. This is due to a timing delay in the system of $1.8 \mu$ s between the pulse generator, modulator, and detector, and has simply been subtracted from all other derived heights. It can be seen that the laser pulse has a form close to a square-wave with a measured width of $150 \mathrm{~m}$, which is what we would expect for a $1 \mu$ s pulse with a rise time of less than $100 \mathrm{~ns}$. For high signalto-noise data, this profile can be used to deconvolve the sodium layer profile to yield a height resolution better than $100 \mathrm{~m}$.

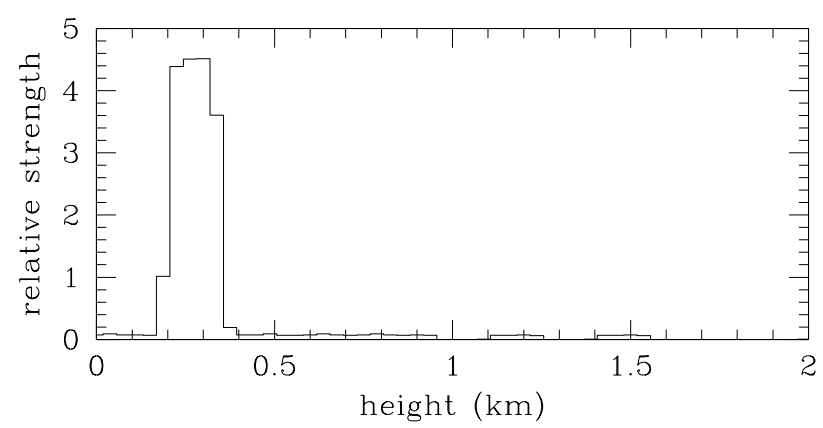

Fig. 2. The profile of the $1 \mu$ s laser pulse, measured by scattering the beam from the telescope dome. The profile is close to being squarewave and has a $F W H M$ of $150 \mathrm{~m}$, as expected. The height offset of $270 \mathrm{~m}$ is due to a delay of $1.8 \mu$ s between the pulse generator, modulator, and detector. It has been subtracted from all subsequent height measurements.

\section{Sodium layer profiling by LIDAR at Calar Alto observatory}

\subsection{Experimental set-up}

\subsubsection{The laser}

The ALFA laser system (Rabien et al. 2000), situated in the Coudé laboratory of the $3.5 \mathrm{~m}$ telescope at Calar Alto Observatory in Spain, has been used to provide an artificial reference star in the mesospheric sodium layer for adaptive optics correction. It could generate a LGS with a magnitude as bright as $V \sim 10$ in good atmospheric transparency. The laser system consisted of a ring dye laser (Coherent 899-21, with some modifications) pumped by a $28 \mathrm{~W} \mathrm{cw}$ argon ion laser (Coherent Innova 400). The output power could exceed $5 \mathrm{~W}$ in single mode with a $10 \mathrm{MHz}$ bandwidth. The frequency was tuned to the sodium $\mathrm{D}_{2}$ line at $589 \mathrm{~nm}$. The laser beam was circularly polarized, pre-expanded, and sent to the $50 \mathrm{~cm}$ diameter laser launch telescope, $2.9 \mathrm{~m}$ from the science telescope optical axis, via a remotely controllable series of relay mirrors (Ott et al. 2000).

\subsubsection{Laser modulation}

An ISOMET 1201E-1 acousto-optical modulator (AOM), driven by a pulse generator (HP 81101A) provided the optical beam modulation. As described in Sect. 2, close to square wave amplitude modulation of the laser beam at $\mathrm{MHz}$ rates was desired to achieve the required height resolution and a maximum returned flux. It is the pulse rise (and fall) time that governs the squareness of a pulse and in the AOM the rise time is dependent on beam diameter. To achieve faster rise times it is necessary to focus the laser beam, so that in the crystal the acoustic transit time across the beam is reduced. The laser modulation set-up is shown in Fig. 3. In order to achieve a pulse rise time below $100 \mathrm{~ns}$, the laser beam was focused by a lens placed about $1 \mathrm{~cm}$ in front of the modulator. The resulting power density of less than $300 \mathrm{~W} \mathrm{~mm}^{-2}$ was well below the limit of $800 \mathrm{~W} \mathrm{~mm}^{-2}$ for the modulator. A second lens approximately compensated for the beam quality degradation 


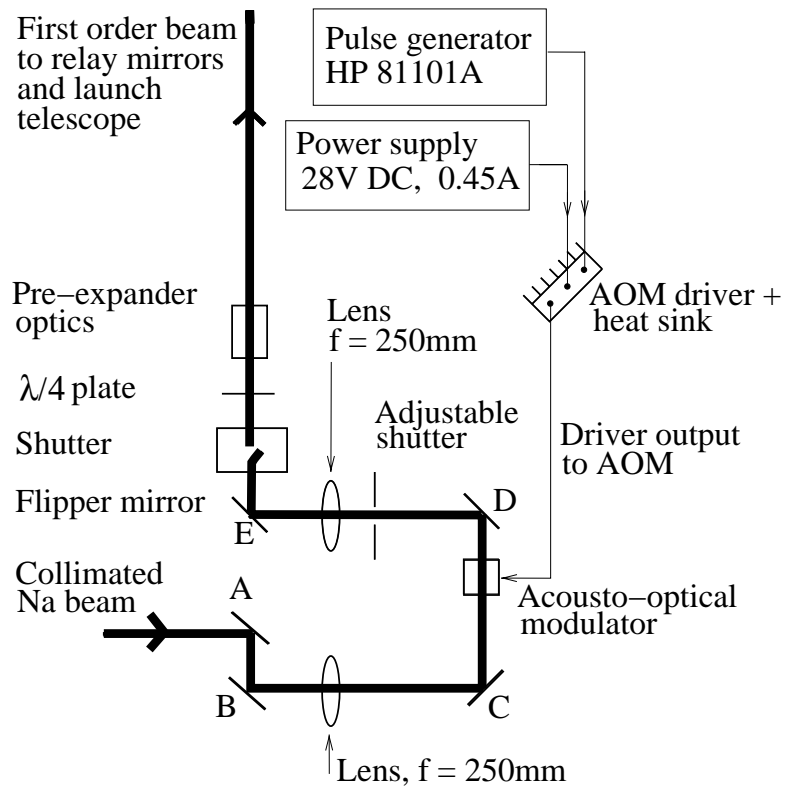

$\mathrm{A}, \mathrm{B}, \ldots ., \mathrm{E}$ : mirrors

Fig. 3. Schematic of the set-up in the laboratory used to modulate the laser beam. The thick line shows the beam path.

introduced by the modulation optics. As seen in Fig. 3, three mirrors steered the beam around the additional loop and then back onto its nominal path. An adjustable shutter placed between the lenses and after the modulator was used to block all spots except the chosen first order spot. Some second order light passed through to the beam expander but this was largely blocked by its beam stop aperture. The first order beam power was maximised by following the recommended procedure: to reduce the driver output power close to its minimum, then find the optimal (i.e. Bragg) angle of the incident laser on the crystal by monitoring an optical power meter, and finally set the driver output power that maximises the first order beam optical power.

A shift in the frequency of the beam arises from modulation of the beam into several orders. Within the modulator, the acoustic waves which create the diffraction pattern that diverts the beam move through the medium, and the frequency of the diffracted beams is doppler shifted with respect to that of the incident beam. If light is incident at the Bragg angle, the frequency shift is $-70 \mathrm{MHz}$ at $\lambda=589 \mathrm{~nm}$. This is an acceptable change because the width of the $\mathrm{D}_{2}$ resonance line is $\sim 1 \mathrm{GHz}$.

We achieved a relatively low modulation efficiency, with only about $70 \%$ of the power transmitted in first order. Including the beam relay transmission, and taking into account the duty cycle of the pulse sequence, the average laser power at the launch telescope was $1.1 \pm 0.1 \mathrm{~W}$ for the experiment.

\subsubsection{Detector set-up}

The accuracy of LIDAR data is limited by the timing resolution of the detector. For this reason CCD detectors are not suitable, and photo-multiplier tubes (PMTs) are more commonly used. However, PMTs have a low quantum efficiency
$(Q E \sim 10 \%)$ and instead we have used an actively quenched silicon Avalanche PhotoDiode (APD) from Perkin Elmer with a $Q E$ greater than $70 \%$. Actively quenched APDs minimize the dead time after each detected photon to $\sim 20 \mathrm{~ns}$. Statistical simulations show that we only expect a maximum of one to two photons from the laser beacon to be detected every microsecond. Therefore, the dead time does not affect the measurements. The APDs have no readout noise and the dark counts are $<1000 \mathrm{~s}^{-1}$; the dominant source of noise is background counts, including sky counts and stray light around the sodium line transmission filter in the optical path in ALFA. In addition to the usual dust and light shields surrounding the bench, heavy black cloth was attached to ALFA to block stray external optical light from reaching the APD. However, much more serious was the presence of infrared LEDs inside the ALFA bench, on motor encoders for example. Although every effort was made to remove these the overall background count rate was 7000-8000 s ${ }^{-1}$.

In mounting the detector on the telescope we only had access to the $\mathrm{F} / 25$ focus close to the wavefront sensor, at which the plate scale is $0.42 \mathrm{~mm} \mathrm{arcsec}{ }^{-1}$. A $12 \times$ magnification of the image of the APD onto the $\mathrm{F} / 25$ focus using a lens was required to obtain a $6^{\prime \prime} \mathrm{FoV}$ (to match the typical size of $<3$ " for the LGS). Given a $200 \mu \mathrm{m}$ diameter APD active area, the plate scale at the APD was therefore $\sim 0.03 \mathrm{~mm} \mathrm{arcsec}^{-1}$. Alignment of the optical system was performed using a white fibre source placed at the $\mathrm{F} / 10$ telescope focus to simulate the laser guide star. As seen in Fig. 4, the beam could be directed either to the WFS or the APD by a mirror on a remotely movable translation stage. Note that this experimental set-up differs significantly to that proposed for the VLT, in which the $2 \%$ of light leaking through one of the mirrors in the AO system will be used for the LIDAR measurements.

\subsubsection{Data collection}

The APD module outputs a TTL pulse every time a photon is received. A similar TTL sync signal is produced from the signal generator at the start of every $16 \mathrm{k}$ bit pseudo-random sequence (the sequence provided with the pulse generator was in fact a $4 \mathrm{k}$ bit sequence repeated 4 times). These two signals are received by a multichannel scaler (MCS) where timing information is recorded. The MCS card (Fastcomtec MCD-2) consists of $128 \mathrm{k}$ channels, each of which corresponds to a $250 \mathrm{~ns}$ interval. When the sync pulse is received the MCS starts at the first channel, and increments its value every time it receives a count from the APD during the subsequent $250 \mathrm{~ns}$. After this time it moves to the next channel and again counts the number of pulses coming from the APD. This procedure continues until the next time a sync pulse is received, i.e. when the MCS starts from the first channel again. In this experiment we oversampled, with a time interval per channel one quarter of a pulse length.

The MCS has a dead time between channels of $0.5 \mathrm{~ns}$. For $3.5-\mathrm{m}$ and larger telescopes this is not significant for the APD channel as it has the same effect as reducing the collecting area of the telescope by less than $0.2 \%$. Its effect can 


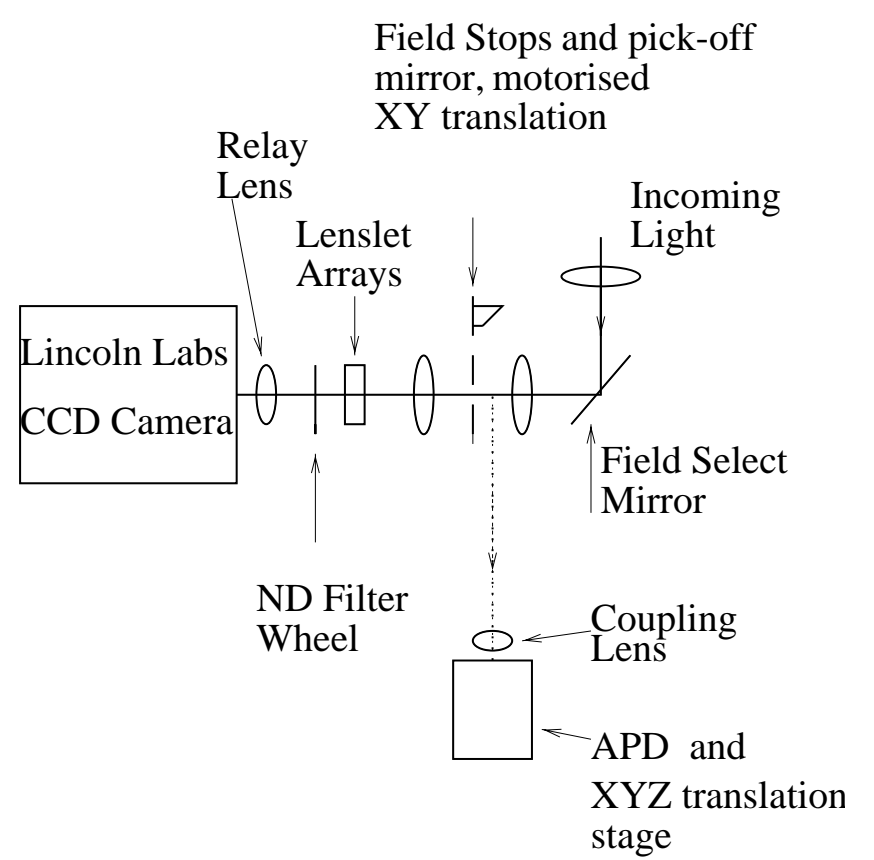

Fig. 4. Schematic of the set-up of the APD LIDAR detector close to the Shack-Hartmann wavefront sensor in ALFA. No neutral density (ND) filter was used during LGS LIDAR observations.

occasionally be seen in the sync channel, and this is why we have twice as many channels as should ever be needed: occasionally the MCS will miss the sync pulse and the collected data will "overflow" into these other channels. Although this data is not used, this precaution prevents the rest from being corrupted.

\subsection{Laser star acquisition}

A 2D detector is required for time efficient laser spot acquisition. Prior to the observations, the APD was aligned to the white light reference beam by adjusting the APD stage to maximize the count rate on the APD event counter. At the beginning of the observations, the laser was pointed to its nominal position on the WFS. One of the white light fibre spots on the WFS was then marked and the corresponding sodium laser WFS spot was moved to this mark. Following this, a pick-off mirror was moved to direct the LGS beam to the APD (Fig. 4), and integration was started.

\subsection{Sodium profiles from LIDAR}

The first measurements using this LIDAR technique with ALFA were obtained in 1999 on October 17 and 18 under extremely poor weather conditions. On the first night the seeing was $4^{\prime \prime}$ or more. Even though the laser had been launched with a $15 \mathrm{~cm}$ beam, in such bad seeing this is much more than a few times the Fried parameter, $r_{0}$, and so the size of the LGS is affected. The final size of the LGS as seen from the ground was at least $6^{\prime \prime}$. As a result, significant light was lost from the 6 " field of view (FoV) of the APD. On the second night there were thin cloud layers at $6 \mathrm{~km}$ and $9 \mathrm{~km}$ (observed
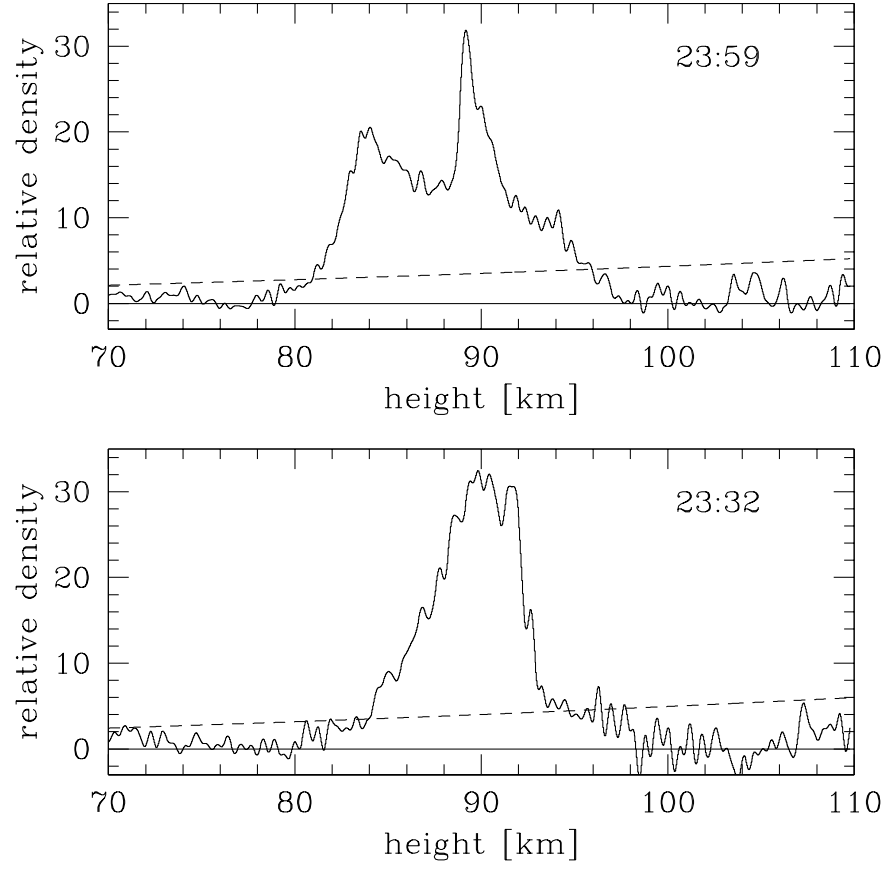

Fig. 5. Examples of profiles of the sodium layer taken on two consecutive nights: Oct. 17 (upper) and Oct. 18 (lower). The UT start time of each $30 \mathrm{~s}$ integration is shown. The $3 \sigma$ noise level (dependent on height) is shown as the dashed line; details of how it is determined are given in the text. Heights are given in $\mathrm{km}$ above Calar Alto Observatory which is itself at $2.2 \mathrm{~km}$. The difference in profile between the two nights is substantial.

by the LIDAR system from the light they scattered). LIDAR integrations were UT time-tagged for comparison with sodium layer profiles derived from direct imaging at a second telescope (see Sect. 3.4). Simultaneous observations of the LGS from this second telescope showed that the observed flux was reduced by a factor of thirty by these cloud layers. The only effect this has on LIDAR is to reduce the signal-to-noise and invalidate absolute flux calibration; the height and profile information (which arise solely from time-tagged data) are unaffected, and thus the first results, shown in Fig. 5 are very encouraging. These data have been convolved with a low-pass digital filter to give a smoothing of $500 \mathrm{~m}$, and only the range encompassing the mesosphere plotted. Additionally, the total flux detected has been normalised for each night, a process which is not normally necessary but which we use in this case because of the significant flux variations due to the clouds.

The Rayleigh cone, which is bright at altitudes less than $\sim 20 \mathrm{~km}$ was not detected. This is because we had a FoV of $6^{\prime \prime}$ and launched the laser $2.9 \mathrm{~m}$ off-axis from the main telescope, which means we could only observe heights in the range $90 \pm$ $30 \mathrm{~km}$. The reason we were able to observe cloud layers below $10 \mathrm{~km}$ is simply because there was so much scattered light from the defocused image of the pupil on these layers, that we could detect it about 1 arcmin away.

The noise is strongly dependent on height, because of the necessary scaling described in Sect. 2. It is determined from the data before the scaling is applied (i.e. while the noise is independent of height) and then scaled in the same way as the 

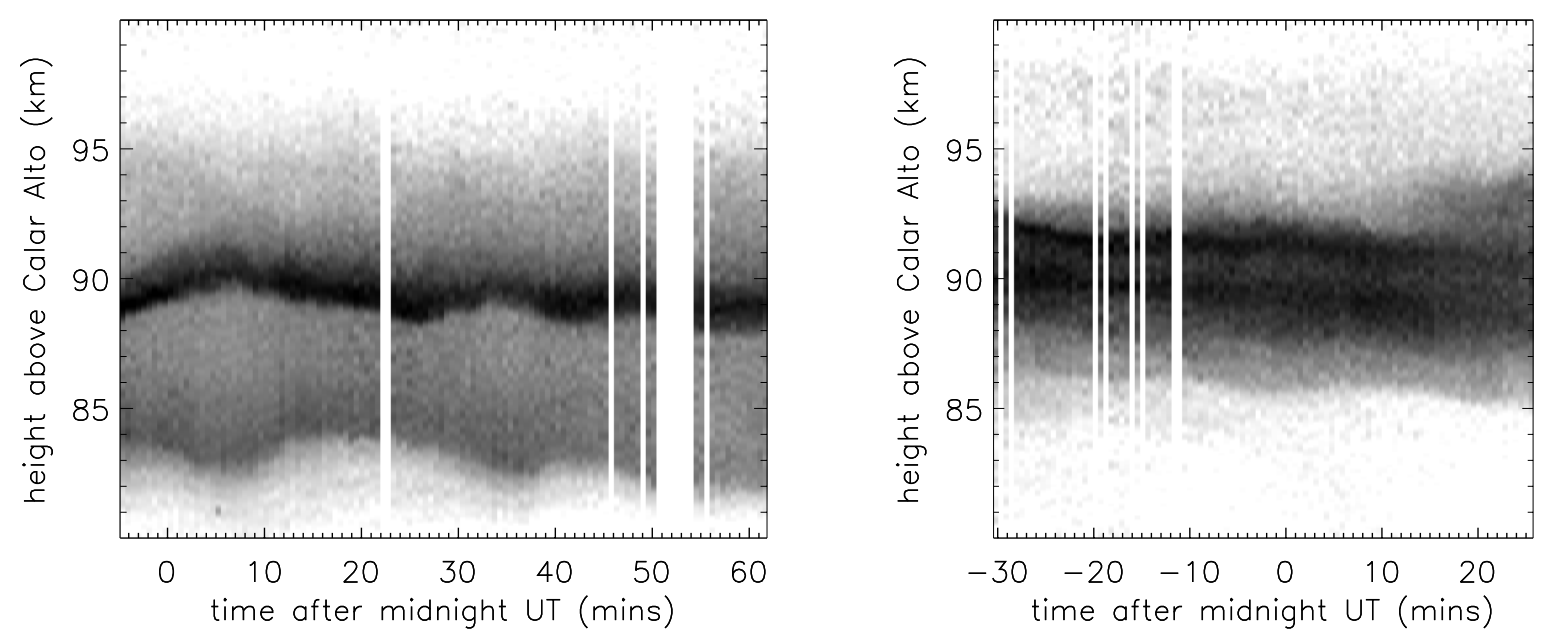

Fig. 6. Density profiles of the sodium layer on Oct. 17 (left) and Oct. 18 (right) 1999, taken every 30 s over a period of 1 hour each night. Darker regions denote a higher atomic density. Blank columns indicate where no data was collected due to clouds.
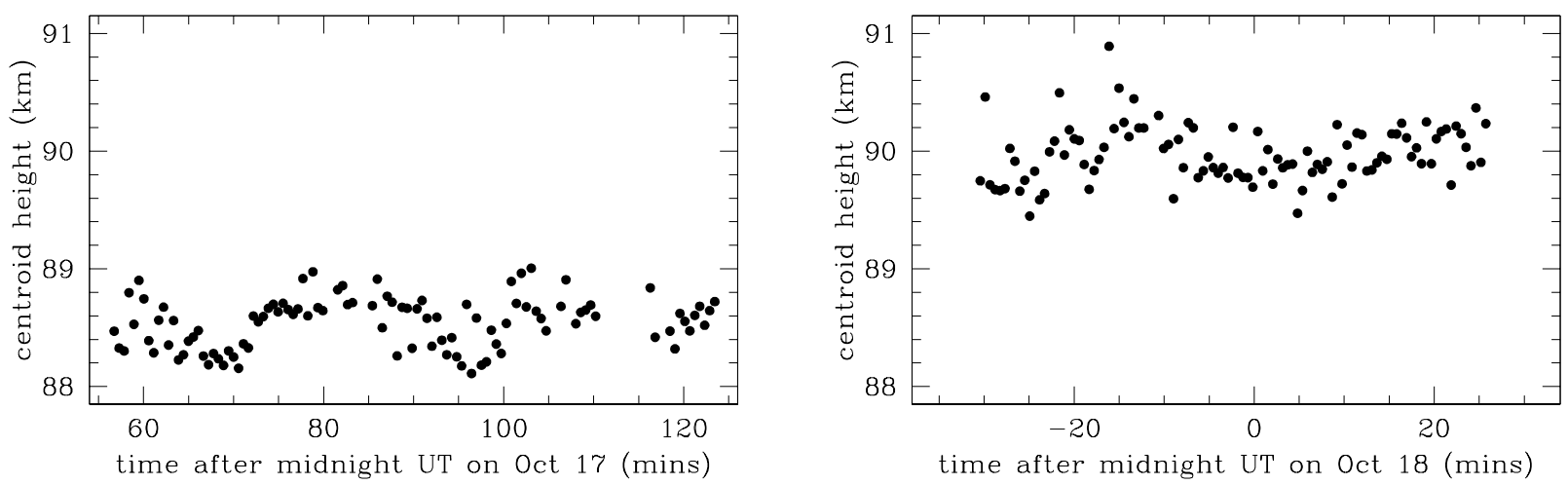

Fig. 7. Plots of centroid height of the sodium layer over time on Oct. 17 (left) and Oct. 18 (right) 1999. Heights are given in km above Calar Alto Observatory which is itself at $2.2 \mathrm{~km}$. There was a $1.5 \mathrm{~km}$ difference in height between the two nights, and further smaller variations during each night.

data - that is, multiplied by the square of the height. The result is that the noise at $100 \mathrm{~km}$ is $25 \%$ more than (and at $80 \mathrm{~km} 20 \%$ less than) that at $90 \mathrm{~km}$. The blank region of the profile used to make the estimate is at $10-60 \mathrm{~km}$, which is large enough that both the photon and correlation noise are implicit in the estimate.

\subsubsection{Results and discussion}

It can be seen from Fig. 6 that using this method we are able to track changes in the sodium layer structure on timescales as short as a few minutes. The two important quantities for adaptive optics are the centroid height and the width over which the layer is spread.

The intensity-weighted centroid height of the atoms shown in Fig. 7, suggests that general trend is for variations of order $500 \mathrm{~m}$ on timescales of $10 \mathrm{~min}$. It is not clear from these data whether the changes between individual points are real effects or a result of the noise in the data (due to the poor conditions under which the experiment was performed). Comparison with the variations in centroid height derived from direct imaging by O'Sullivan et al. (2000) at the same observatory during 1998 would suggest that these could indeed be real variations.
Additionally, there is no correlation between properties of the layer between the two nights. On Oct. 18, the layer (excluding the sporadic) had a distribution which appears almost bimodal, with a centroid height of $92 \mathrm{~km}$ above mean sea level; on Oct. 17, the centroid height was only $91 \mathrm{~km}$, and there was a very prominent narrow peak. Because it had a FWHM of only a few hundred metres and moved independently from the rest of the layer, we identify this as a sporadic layer. Within the sporadic layer, the sodium density is about twice that of the underlying profile, similar to the factor of two to three observed during the O'Sullivan et al. (2000) direct imaging observations.

The FWHM of the layer also shows significant changes. On Oct. 17, the layer had a $F W H M$ of around $12-13 \mathrm{~km}$; on Oct. 18 this had reduced to only $5-7 \mathrm{~km}$. This has important consequences on the elongation of the LGS spot as seen from different portions of the science telescope primary mirror, and hence on the performance of an AO system correcting on the LGS. For a laser projected from the side of a 10-m primary mirror, as occurs at the Keck telescope, this can result in elongations in the range 1.5-3.1" for apertures located on the far side of the pupil. On nights when the layer has a large FWHM, the performance of an AO system correcting on the elongated LGS will be compromised; not only due to the shape 
of the spot, but also because it may be truncated by the limited aperture of the wavefront sensor, and also due to the reduced signal to noise in the measurement of the spot positions. At the VLT and Gemini, it the laser will be projected from behind the secondary mirror. This reduces the maximum elongation to the range $0.6-1.3^{\prime \prime}$ for the cases above. When convolved with both the seeing and the intrinsic size of the LGS (combined, about $1.2^{\prime \prime}$ ), this has only a small effect on the shape of the LGS.

\subsection{Comparison to direct imaging}

In order to validate the profiles obtained using the LIDAR method, we simultaneously observed the LGS plume from another telescope. For this we used the 2.2-m telescope, separated from the $3.5-\mathrm{m}$ by $260 \mathrm{~m}$. The detector was a $2048 \times 2048$ pixel SITE\#1d CCD camera with a pixel scale of $0.53^{\prime \prime}$, installed at its Cassegrain focus. A narrow band interference sodium filter was used to minimize the background. Images, each a $30 \mathrm{~s}$ exposure, were time-tagged with the UT start time. The purpose of the observations was multifold. The monitoring of the sodium spot was meant to detect possible sporadics and determine the centroid height variation. The hope was to achieve photometric study of the Rayleigh scattering and sodium spot. Therefore spectrophotometric standards have been observed regularly. Unfortunately the weather conditions were not photometric and the results can therefore only be indicative. Here we consider only a comparison with the LIDAR data for which, to ensure it is meaningful, only images acquired within $30 \mathrm{~s}$ of LIDAR data are used.

Since no sky frames were acquired, no sky subtraction could be applied. Only a dark of equivalent integration time was subtracted before flat-fielding with a dome flat. Bad pixel correction was also applied. In a cosmetic final stage, star trails crossing the plume or Rayleigh beam were removed by thresholding the data. Figure 8 illustrates a typical result.

Due to the geometrical situation of the telescopes and the fact that the altitude is aligned with the horizontal axis in the images, the altitude of a point can be determined as:

Altitude $=z_{0}+\left[d * \tan \left(\alpha-\left(x_{0}-x\right) *\right.\right.$ pixscale $\left.)\right]$,

where $z_{0}$ is the altitude of the observing site $(2.2 \mathrm{~km}), d$ the distance between the telescopes, $\alpha$ the elevation of the 2.2-m telescope, and pixscale is the pixel scale of the camera used. $x_{0}$ has been chosen as the middle of the CCD FoV. Using this formula, we noticed a difference in altitude, and altitude range, between the imaged and LIDAR profiles. To obtain a similar altitude range for both sets of data, the 2.2-m pointing had to be considered 7.12 arcmin higher than that actually indicated at the telescope. This can be attributed to the fact that (a) the 3.5-m (and laser) was pointing to nominal, but not necessarily actual, zenith; (b) for observations of the LGS, the 2.2-m pointing was measured as an angular offset from nominal zenith which again may not necessarily be actual zenith; and (c) there was a repeated offset of nearly 6 arcmin from the CCD frame centres when pointing to calibration stars, suggesting a large error in the pointing model of the telescope.
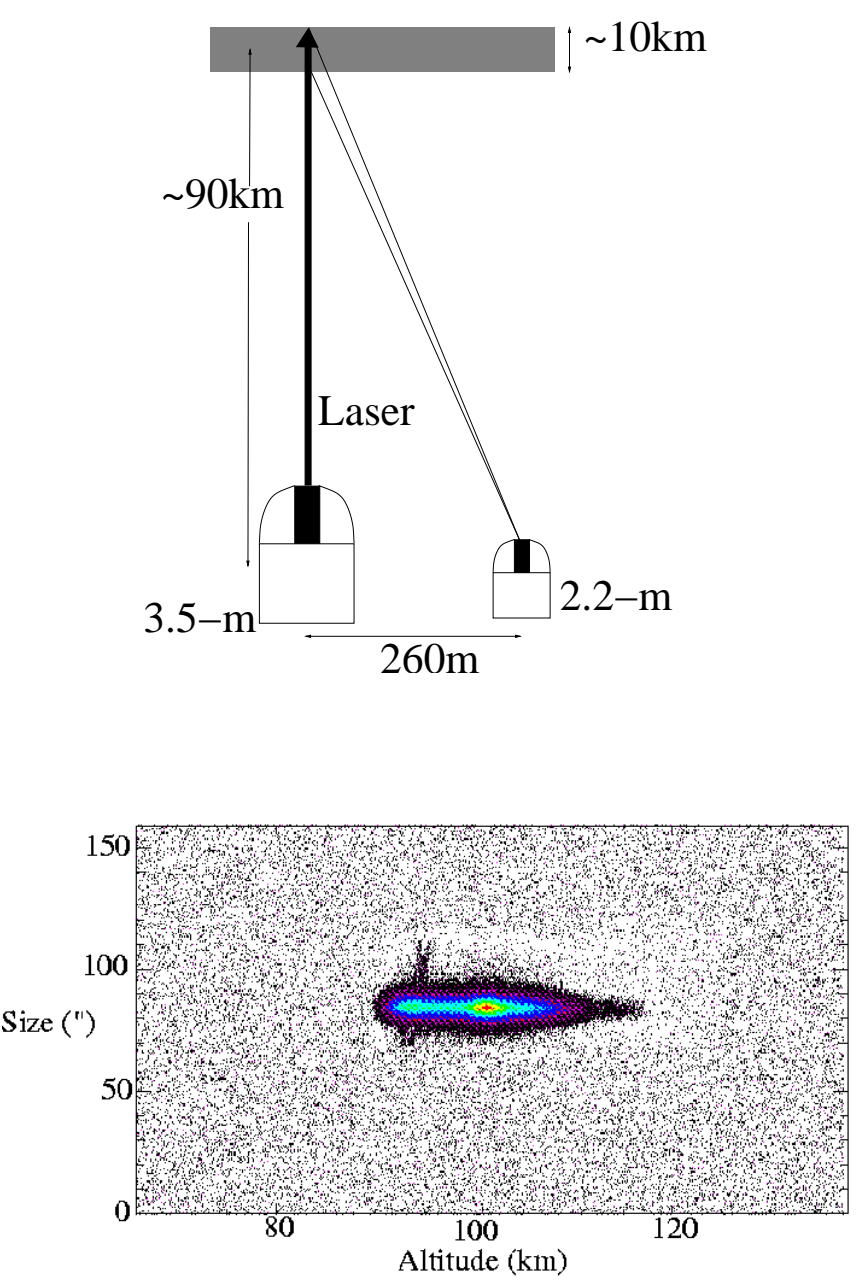

Fig. 8. Top: sketch showing the geometry of the laser guide star projected from the $3.5-\mathrm{m}$, and the direct imaging observations from the $2.2-\mathrm{m} 260 \mathrm{~m}$ away. Bottom: typical image of the ALFA LGS plume as observed from the 2.2-m telescope. See Sect. 3.4 for details of the data reduction.

Once the data was calibrated in altitude, a scaling calibration was applied: the integrated flux of the 2.2-m data between 70 and $110 \mathrm{~km}$ was forced to equal the integrated flux of the LIDAR profile in the same altitude range.

Profiles were obtained by summing rows of 10 pixels perpendicular to the altitude axis. Figure 9 shows that a very good agreement can be found between the data originating from the two different observing techniques. In these data CCD imaging of the sodium layer appears to give profiles with better signalto-noise than the LIDAR technique, but this is likely to be due to a reduced count rate in the APD FoV either resulting from the poor seeing or problems with alignment. The effect of the poor seeing is certainly apparent in the amplitude of the sporadic layer: it reduced the height resolution of the imaging data to approximately $1 \mathrm{~km}$, far inferior to the $150 \mathrm{~m}$ attainable with LIDAR. The discussion above also confirms that LIDAR is superior in terms of absolute height calibration. 

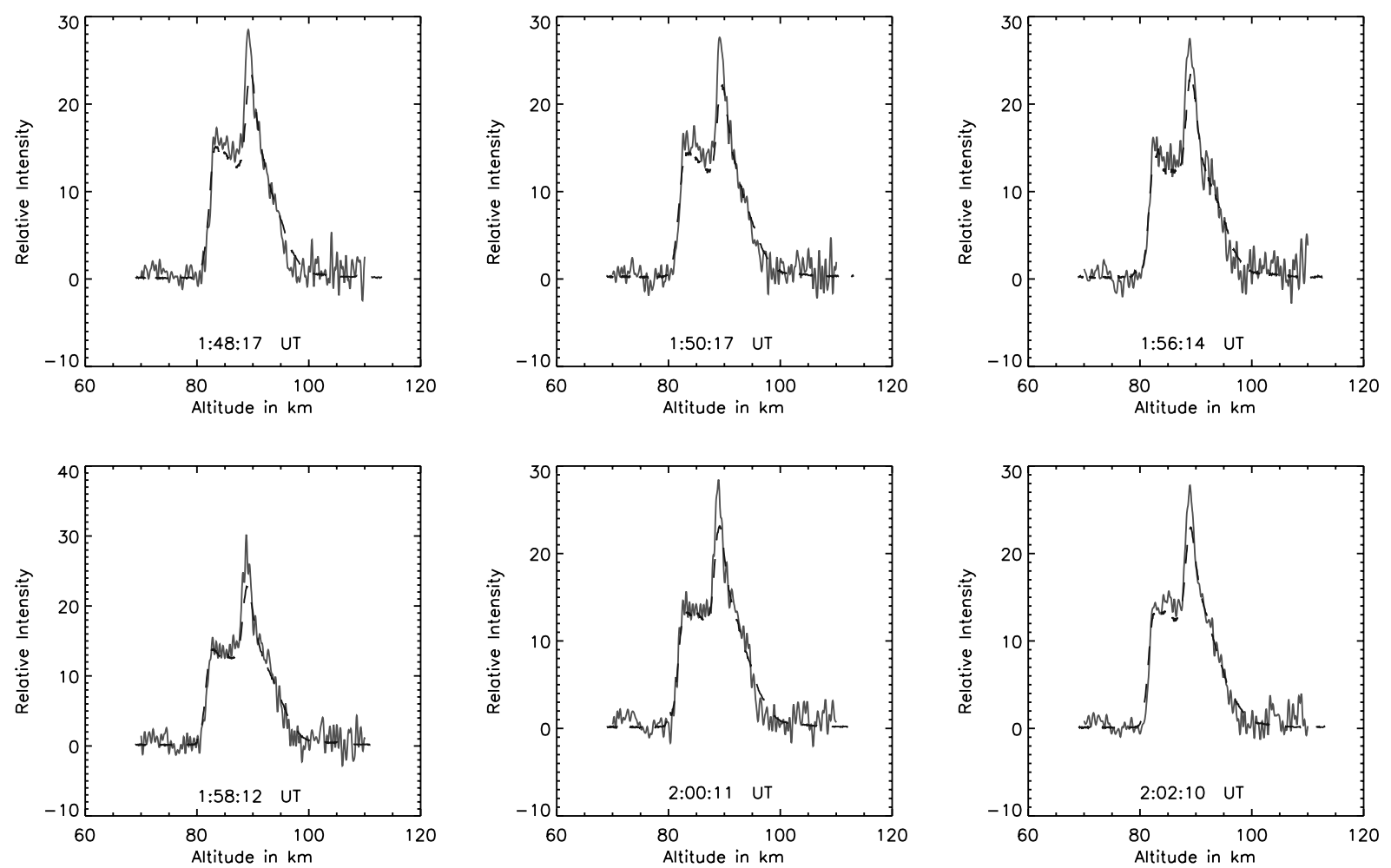

Fig. 9. Results of the sodium profile on Oct. 17 derived from the LIDAR experiment (solid line), compared to the direct imaging results (dashed line).

\section{Expected performance of the VLT sodium profiler}

\subsection{Some considerations}

The concept of the VLT sodium profiler (Butler et al. 2002) is the same as that built for ALFA. The differences are mostly in hardware in the sense that the profiler is more automated and will integrate well into a VLT instrument control system. Additionally, the VLT profiler will have a longer $32 \mathrm{k}$ bit pulse sequence mainly for lower cross-correlation noise. Below, we summarize the salient points learned from the ALFA LIDAR experiment, and from signal-to-noise calculations for the VLT sodium profiler.

Firstly, laser pulse length affects height resolution and signal-to-noise. A shorter pulse length allows a higher resolution in the sodium layer profile measurement: $1 \mu$ s corresponds to $150 \mathrm{~m}, 2 \mu$ s to $300 \mathrm{~m}$, etc. However, there are advantages to a longer pulse: it gives better signal-to-noise in the profile and a more accurate mean height measurement than can be achieved simply by binning the data afterwards. It will be possible to adjust the pulse length in the VLT LGS LIDAR system over the range $0.1-10 \mu \mathrm{s}$. It is assumed that the data is collected over time periods equal to half the pulse length.

Secondly, there is the issue of noise. The basic profile recovered by LIDAR is the apparent distribution from which the photons originate, and in this profile the noise is independent of height. To derive the sodium atom density distribution, each point in this basic profile must be multiplied by the square of its distance. As a result, the noise in the density profile increases quadratically with distance.
Thirdly, we should ask what is the most sensible average height calculation. The most obvious method to find the average height of the sodium layer is to calculate the centroid height of the atoms in the layer, as we did for the ALFA LIDAR experiment. But this has an intrinsic bias and the value calculated actually depends on the resolution used. An alternative is to use the median height of the atoms, which is independent of the resolution (at least with pulses of up to $4 \mu \mathrm{s}$ ) as long as the profile changes only slowly around the $600 \mathrm{~m}$ either side of the median height; linear interpolation can be used between these points. However, the WFS detects photons, and so instead the apparent distribution from which the photons originate should be used. This differs from the actual density distribution: for a uniform sodium atom distribution, $25 \%$ fewer photons will be detected from a distance of $95 \mathrm{~km}$ than from $85 \mathrm{~km}$. Probably the best method is to find the height above which (and below which) half the sodium-line photons originate. In this paper, it is called the "median flux height".

Finally, there may be a systematic height offset. With the LIDAR system on ALFA, there was a large constant offset of $270 \mathrm{~m}$ in the height measurement. This is likely also to occur with the VLT LGS system, but can likewise easily be calibrated by performing a LIDAR measurement with a reflective layer (such as the observatory dome) at almost zero height.

\subsection{Simulations}

Simulations of LIDAR have been carried out to look at the signal-to-noise issues and determine quantitatively what are reasonable integration times, pulse lengths, etc. The uncertainties in average height have been estimated from running 100 simulations for each parameter set. 

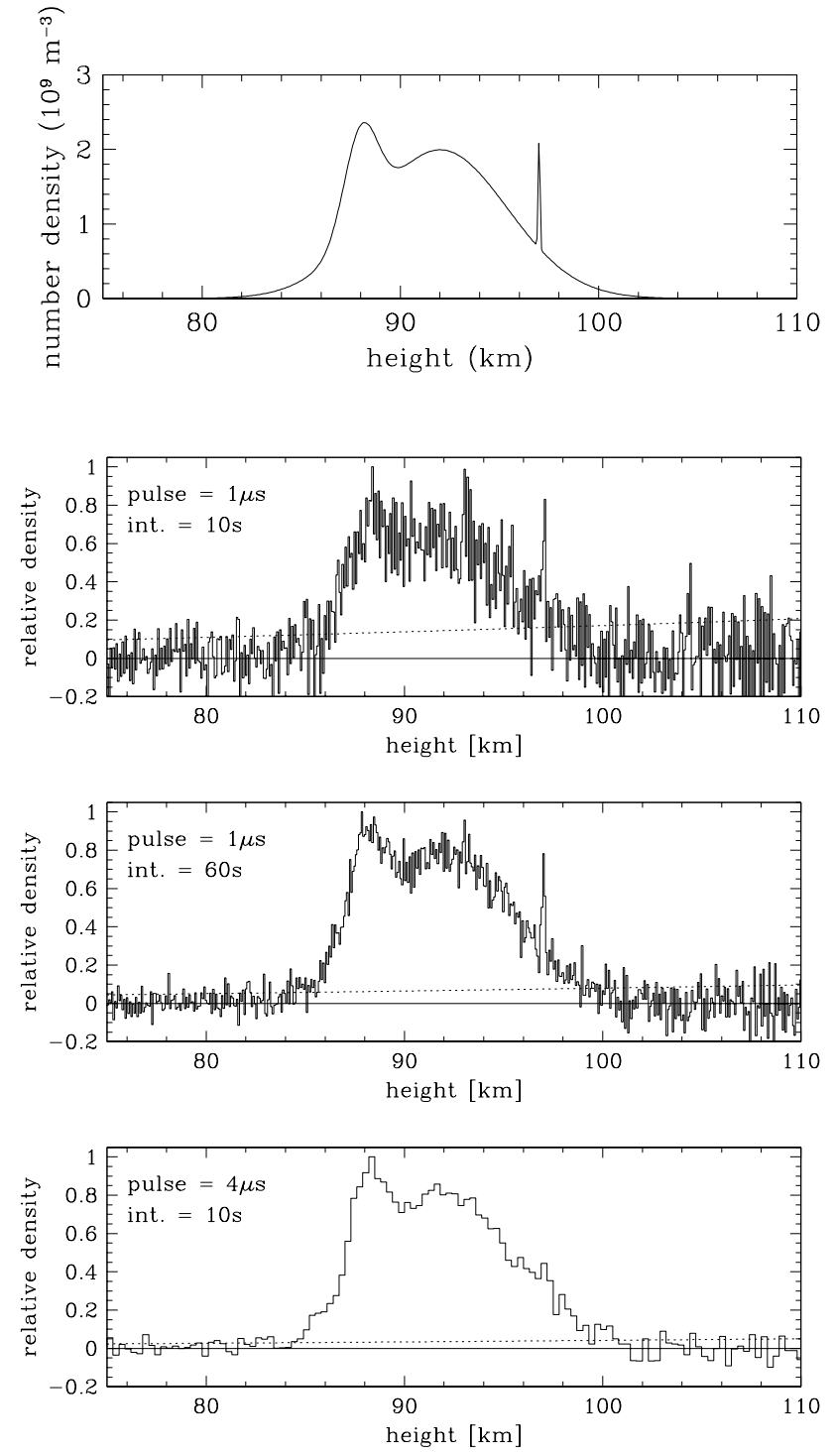

Fig. 10. Top panel: the model sodium layer used in the LIDAR simulations. Lower three panels: recovered profiles for different pulses widths and integration times.

The input to the model was the sodium density distribution shown in the top panel of Fig. 10. To indicate the difference in the "average" height obtained when it is calculated in different ways, these are given for this profile as a reference: $91.52 \mathrm{~km}$ (centroid height); $91.33 \mathrm{~km}$ (median height); and $91.01 \mathrm{~km}$ (median flux height). The difference between the centroid and median heights of the atoms is $200 \mathrm{~m}$; that between the median height of the atoms and the median height from which the photons originate is an additional $300 \mathrm{~m}$. Since the acceptable error (Kissler-Patig 2001) is only $200 \mathrm{~m}$ at zenith it is important to use the correct height estimator.

It has been assumed in the simulations that the APD will be mounted in one of the AO systems at the VLT with an effective pupil diameter of $4.0 \mathrm{~m}$, which would provide a field of view of $3^{\prime \prime}$. The collector transmission in the simulation is $1 \%$ (which includes the $2 \%$ through a mirror in the $\mathrm{AO}$ system). It has been assumed that transmission of the upward beam through the LIDAR optics (in particular the modulator) is $50 \%$, so that
Table 2. Parameters used in the simulations of the VLT profiler.

\begin{tabular}{ll}
\hline \hline sodium column density & $2 \times 10^{13} \mathrm{~m}^{-3}$ \\
atmospheric transmission & $75 \%$ \\
laser power (during a pulse) & $5 \mathrm{~W}$ \\
pulse length & $1 \mu \mathrm{s}$ \\
launch transmission & $60 \%$ \\
collector transmission & $1 \%$ \\
zenith distance & $0^{\circ} / 60^{\circ}$ \\
integration time & $30 \mathrm{~s} / 60 \mathrm{~s}$ \\
\hline
\end{tabular}

Table 3. Standard deviation, $\sigma_{\mathrm{H}}$, for the median flux height for $30 \mathrm{~s}$ and $60 \mathrm{~s}$ integrations at zenith distances of $0^{\circ}$ and $60^{\circ}$. A pulse length of $1 \mu \mathrm{s}$ has been used.

\begin{tabular}{lll}
\hline \hline $\begin{array}{l}\text { Integration time } \\
(\mathrm{s})\end{array}$ & $\begin{array}{l}\sigma_{\mathrm{H}}\left(z=0^{\circ}\right) \\
(\mathrm{m})\end{array}$ & $\begin{array}{l}\sigma_{\mathrm{H}}\left(z=60^{\circ}\right) \\
(\mathrm{m})\end{array}$ \\
\hline 30 & 47 & 318 \\
60 & 39 & 199 \\
\hline
\end{tabular}

even during an "on" pulse the effective laser power is only $5 \mathrm{~W}$. This is likely to be an underestimate. The important parameters used in the simulation are given in Table 2.

The simulation is in two parts. One calculates the return flux as a function of time measured by the MCS. So that the data can be sampled twice for each pulse, each digit of the pulse sequence is repeated (and the associated time-step halved). The data for the sodium layer are then binned appropriately, and convolved with the double-length pulse sequence. The resulting vector is scaled so that it correctly reflects the average flux over one sequence. At this stage photon noise, background noise, and dark counts are added. This is repeated and the results co-added until the required integration time is reached. This represents the signal detected by the MCS. The second part of the code takes this "observed" data and calculates what the sodium profile was. It is based on that used during the ALFA LIDAR experiment, and is effectively a convolution of the pulse sequence (padded with an extra zero after each digit) and the MCS data. A background is then subtracted, the noise estimated, and the height scaling imposed.

A set of three typical profiles are shown in the lower three panels of Fig. 10; the central one of these corresponds to the $60 \mathrm{~s}$ integration time at zero zenith distance in Table 3. These demonstrate the effect on the profile of varying the integration time and pulse length. In each of these, the dotted line marks the $3 \sigma$ background noise level.

Table 3 shows the standard deviation (in metres) for the median flux height, at zenith distances of $0^{\circ}$ and $60^{\circ}$, and for $30 \mathrm{~s}$ and $60 \mathrm{~s}$ integrations. The 3 -sigma uncertainty at zenith is $150 \mathrm{~m}$ for a $30 \mathrm{~s}$ integration, below the requirement of $200 \mathrm{~m}$. The simulations assume a relatively low sodium column density. Under conditions worse than this, it may not be possible to observe using a LGS. However, if a profile of the sodium layer were required, it would be possible to reduce the error by as much as a factor two by increasing the pulse length to $2-4 \mu \mathrm{s}$. 


\section{Conclusion}

1. We have developed and tested a method, based on LIDAR, of measuring the height and profile of the mesospheric sodium layer using a cw laser. This avoids the need for a second telescope, has almost no dependence on the atmospheric conditions, and has a very small error in the absolute height. The method is designed to be used at observatories with a LGS AO system, and can be performed using the same laser during such observations.

2. We have tested our method with an experiment using the ALFA laser at Calar Alto observatory in Spain. The profiles derived from the LIDAR measurements compare well to those obtained by simultaneous direct imaging and confirm that the method can be used successfully.

3. The profiles we measured during this experiment demonstrate clearly that there is a need to measure changes in sodium layer height in order to set the initial wavefront sensor focus prior to, and maintain the correct focus during, observations. The difference in centroid height on consecutive nights was $1.5 \mathrm{~km}$, and changes of several hundred metres were measured on timescales of a few minutes. The data also show large changes in the $F W H M$ of the layer, which can have consequences for the apparent elongation of the LGS spot as seen by a wavefront sensor.

4. We have simulated the performance of a system to be integrated into the VLT Laser Guide Star Facility. This has highlighted the importance of choosing the correct method of deriving the "average" distance to the sodium layer, and we propose that the "median flux height" is the appropriate estimator. A $3 \sigma$ error of less than $200 \mathrm{~m}$ can be achieved with only a $30 \mathrm{~s}$ integration under the required conditions.

Acknowledgements. DB, RD, HF and NA acknowledge funding from the TMR European Network for Laser Guide Stars at $8 \mathrm{~m}$ Class Telescopes under contract ERBFMRXCT 960094. DB acknowledges the support of the research and training network on "Adaptive Optics for Extremely Large Telescopes" under contract HPRN-CT-2000-00147. It is a pleasure to thank Wolfgang Hackenberg, Jesus Aceituno, Robert Weiss and the technical staff at MPIA for valuable assistance during the experiment set-up. $\mathrm{S}$. Hippler is thanked for help in preparing the colour pulse pattern figure. It is a pleasure to thank the anonymous referee for a valuable comment.

\section{References}

Ageorges, N., \& Hubin, N. 2000, A\&AS, 144, 533

Bonaccini D., et al. 2002, in Adaptive Optical System Technologies II, ed. P. L. Wizinowich, \& D. Bonaccini, SPIE, 4839
Bonnet, H. 2001, in SINFONI AO-Module: Control of the Trombone position in LGS mode, ESO document VLT-TRE-ESO-14710-2598, issue 1.0

Butler, D. J., Hippler, S., Neumann, U., et al. 2002, in Adaptive Optical System Technologies II, ed. P. L. Wizinowich, \& D. Bonaccini, SPIE, 4839

Clemesha, B. R., Simonich, D. M., Batista, P. P., \& Kirchhoff, V. W. J. H. 1982, J. Geophys. Res., 87, 181

Clemesha, B. R. 1995, J. Atmos. Phys., 57, 725

Collins, S. C., Plane, J. M. C., Kelley, M. C., et al. 2002, J. Atmos. Sol. Terr. Phys., 64, 845

Davies, R., Eckart, A., Hackenberg, W., et al. 2000, Expt. Ast., 10, 103

Davies, R. I., Ott, T., Li, J., et al. 2002, in Adaptive Optical System Technologies II, ed. P. L. Wizinowich, \& D. Bonaccini, SPIE, 4839

D’Orgeville, C., Rigaut, F., Boccas, M., et al. 2002, in Adaptive Optical System Technologies II, SPIE, 4839

Foy, R., \& Labeyrie 1985, A\&A, 152, L29

Gardner, C. S., Kane, T. J., Hecht, J. H, et al. 1991, Geophys. Res. Lett., 18, 1369

Ge, J., Jacobsen, B. P., Angel, J. R. P., et al. 1998, in Adaptive Optical System Technologies, ed. D. Bonaccini, \& R. K. Tyson, SPIE, 3353, 242

Hecht, J. H., Kane, T. J., Walterscheid, R. L., et al. 1993, J. Atmos. Terr. Phys., 55, 409

Kasper, M., Looze, D., Hippler, S., et al. 2000, Exp. Ast., 10, 49

Kissler-Patig, M. 2002, in AO Top Level Requirements for the LGSF, ESO document VLT-SPE-ESO-11600-2369, issue 2.2

Kwon, K. H., Senft, D. C., \& Gardner, C. S. 1988, J. Geophys. Res., 93, 14199

Le Louarn, M. 2000, Ph.D. Thesis, Univ. Lyon

Megie, G., Bosk, F., \& Blamont, J. E. 1976, Planet. Space Sci., 225, 1093

Michaille, L., Canas, A. D., Dainty, J. C., et al. 2000, MNRAS, 139, 318

Michaille, L., Clifford, J. B., Dainty, J. C., et al. 2001, MNRAS, 328, 993

O’Sullivan, C. M. M., Redfern, R. M., Ageorges, N., et al. 2000, Expt. Ast., 10, 147

Ott, T., Hackenberg, W., Rabien, S., et al. 2000, Expt. Ast., 10, 89

Papen, G. C., Gardner, C. S., \& Yu, J. 1996, in Adaptive Optics, Vol. 13, OSA Technical Digest (OSA, Washington DC), 96

Pennington, D., et al. 2002, in Adaptive Optical System Technologies II, ed. P. L. Wizinowich, \& D. Bonaccini, SPIE, 4839

Rabien, S., Ott, T., Hackenberg, W., et al. 2000, Expt. Ast., 10, 76

Rabien, S., Davies, R. I., Ott, T., et al. 2002, in Adaptive Optical System Technologies II, ed. P. L. Wizinowich, \& D. Bonaccini, SPIE, 4839

She, C. Y., Chen, S., Hu, Z., et al. 2000, Geophys. Res. Lett., 27, 3289

Sica, R. J., Thayaparan, T., Argall, P. S., et al. 2002, J. Atmos. Terr. Phys., 64, 915

Simonich, D. M., Clemesha, B. R., \& Kirchhoff, V. W. J. H. 1979, J. Geophys. Res., 84, 1543 